\title{
Effect of Intra-arterial Balloon Pumping for Refractory Septic Cardiomyopathy: A Case Series
}

\author{
Yuji Takahashi ${ }^{1}$, Tomohiro Sonoo ${ }^{2}$, Hiromu Naraba ${ }^{3}$, Hideki Hashimoto ${ }^{4}$, Kensuke Nakamura ${ }^{5}$
}

\begin{abstract}
Background and aims: Patients with septic cardiomyopathy (SCM) occasionally develop refractory cardiogenic shock, which is difficult to resolve even with the administration of standard dose of catecholamines. Although venoarterial extracorporeal membrane oxygenation (VAECMO) has recently been reported with good treatment results, there have been no evidence-based practices. Furthermore, severe SCM may be fatal if the blood pressure cannot be increased. This case series explored whether the application of intra-arterial balloon pumping (IABP) is an effective method for increasing blood pressure in patients with severe SCM.

Subjects and methods: Over a 58-month period, all patients who were admitted in the emergency and critical care center and managed with IABP were investigated. Among these, data sets of patients diagnosed with SCM were evaluated retrospectively.

Results: Ten patients were included in this analysis. Their mean APACHE II and SOFA scores were $26.8 \pm 7.9$ and $13.9 \pm 1.7$, respectively. A mean arterial pressure (MAP) increase of more than $30 \%$ was achieved in six patients, and a decrease in catecholamine index was observed in five. The effective group consisted of seven patients. The stroke volume increased in $83 \%$ of patients who were equipped with pulmonary artery or transpulmonary thermodilution catheter. Low heart rate and regular heart rhythm may be important factors for the effectiveness of IABP for cardiogenic shock caused by refractory SCM.

Conclusion: Intra-arterial balloon pumping may be able to raise MAP in refractory SCM patients even with septic shock by an increase in cardiac output.

Keywords: Cardiogenic shock, Critical care, IABP, Sepsis, Septic cardiomyopathy

Key messages: Intra-arterial balloon pumping for refractory SCM may be an effective method to stabilize circulation status, especially for patients with low heart rates and regular rhythm.

Indian Journal of Critical Care Medicine (2019): 10.5005/jp-journals-10071-23150
\end{abstract}

\section{INTRODUCTION}

Some septic patients have impaired cardiac function without ischemia or cardiomyositis. This pathologic state is called septic cardiomyopathy (SCM) and is a septic organ dysfunction. Although the definition of SCM is obscure yet, some recent studies use echocardiography and serum cardiac biomarkers for diagnosis. ${ }^{1,2}$ A few patients with SCM may sometimes exhibit drug-resistant refractory cardiogenic shock. ${ }^{3-6}$

Similar to the standard management of cardiogenic shock, the administration of dobutamine is recommended for refractory SCM after proper fluid resuscitation and noradrenaline administration. ${ }^{7,8}$ Beta stimulants, which are occasionally used for septic shock, may not be as effective in SCM patients due to the downregulation of beta adrenergic receptors caused by inflammatory mediators., ${ }^{9,10}$ Therefore, a few patients with SCM may ultimately exhibit refractory cardiogenic shock since there are currently no evidence-based methods to achieve target blood pressure. In a recent review article, several methods, including IABP and venoarterial extracorporeal membrane oxygenation (VA-ECMO), are introduced as treatments for their refractory shock. ${ }^{11}$ Although VA-ECMO has recently been reported to have $71 \%$ survival to discharge ${ }^{12}$ for refractory $S C M$, its effectiveness has not yet been established. Moreover, IABP for SCM has not been clarified except for a case report. ${ }^{13}$ Generally, IABP supports cardiac function by two effects: (1) systolic-unloading and (2) diastolic-augmentation, where the former may decrease systolic blood pressure while the latter increases diastolic blood pressure. However, these effects have not been validated in septic

\footnotetext{
${ }^{1-5}$ Department of Emergency and Critical Care Medicine, Hitachi General Hospital, Hitachi, Ibaraki, Japan

Corresponding Author: Yuji Takahashi, Department of Emergency and Critical Care Medicine, Hitachi General Hospital, Hitachi, Ibaraki, Japan, yuji.takahashi.ax@hitachi.com

How to cite this article: Takahashi Y, Sonoo T, et al. Effect of Intraarterial Balloon Pumping for Refractory Septic Cardiomyopathy: A Case Series. Indian J Crit Care Med 2019;23(4):182-185.

Source of support: Nil

Conflict of interest: None
}

patients who may have distributive shock. Theoretically, the application of IABP can either increase or decrease mean arterial pressure (MAP). However, in intensive care unit (ICU) settings, we occasionally experience MAP increase and improvement in systemic perfusion status after IABP insertion in septic shock patients with SCM. ${ }^{13}$ We collected cases of SCM patients who were with refractory cardiogenic shock and treated with IABP in our ICU and evaluated the efficacy of IABP for those patients. Our primary interest is the avoidance of acute death from cardiogenic shock, not accomplishing long-term survival.

\section{Subjects and Methods}

Our medical and surgical ICU is managed by emergency physicians and accepts all patients from the emergency department. The study term was between July 2013 and April 2018, and all patients

(c) The Author(s). 2019 Open Access This article is distributed under the terms of the Creative Commons Attribution 4.0 International License (http://creativecommons. org/licenses/by/4.0/), which permits unrestricted use, distribution, and non-commercial reproduction in any medium, provided you give appropriate credit to the original author(s) and the source, provide a link to the Creative Commons license, and indicate if changes were made. The Creative Commons Public Domain Dedication waiver (http://creativecommons.org/publicdomain/zero/1.0/) applies to the data made available in this article, unless otherwise stated. 
managed with IABP in the ICU were investigated. Among these, data sets of patients with sepsis were extracted and examined retrospectively. In this study, SCM patients were defined as follows: satisfied the sepsis-3 criteria, ${ }^{4}$ required the administration of catecholamines, presence of low visual left ventricular ejection fraction (LVEF) confirmed by bedside-echocardiography, and had no clinical evidence for ischemic heart disease or acute cardiomyositis (e.g. changes in electrocardiography or increases in ischemic markers). SCM and stress-induced cardiomyopathy are difficult to clearly distinguish, since SCM is one of stressinduced cardiomyopathy caused by septic inflammatory response. Therefore, we included the patients as SCM if the patient is with low visual LVEF and without troponin elevation. Patients that were previously treated with VA-ECMO when IABP was initiated, diagnosed with acute myocardial infarction, and did not experience cardiogenic shock were excluded. We diagnosed cardiogenic shock based on the Nohria-Stevenson criteria: the simultaneous occurrence of hypoperfusion and conjunction despite adequate preload as well as results obtained from bedside echocardiography, pulmonary artery catheter, and transpulmonary thermodilution method.

The primary outcomes were as follows: increase in MAP of more than $30 \%$ and decrease in catecholamine dosage as indicated by the catecholamine index (CAI). CAI was calculated as follows: CAI $=$ dopamine dose + dobutamine dose + (noradrenaline dose + adrenaline dose) $\times 100(\mu \mathrm{g} / \mathrm{kg} / \mathrm{min})$. The effective group was defined as the group of patients who achieved at least one of the primary outcomes. In our ICU, the vital sign data are taken every 2 hours, and the change of catecholamine doses is immediately transcribed in the chart. Thus, we decided to measure the outcome by referring the vital signs and catecholamine data which were described right after IABP initiation. The secondary outcomes were the survival rates on the 3rd, 7th, and 28th day after IABP initiation, changes in heart rate and stroke volume, and rate of VA-ECMO escalation. Statistical analysis was not performed due to the small sample size. The outcomes were analyzed by calculating the mean and SD of each parameter. This evaluation was approved by the Research Ethics Committee of Hitachi General Hospital (2013-2048).

\section{RESULTS}

During the study period, 38 patients were treated with IABP in our ICU. Among these, 28 were excluded: 25 did not meet the inclusion
$38 \mathrm{IABP}$ use in the ICU during the evaluation period

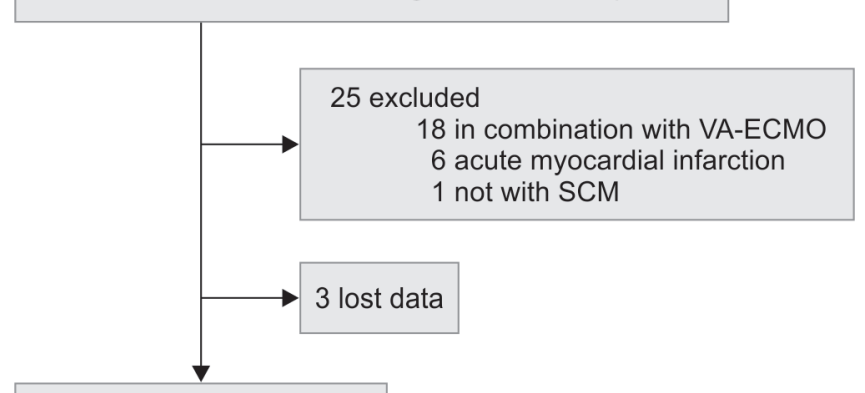

10 included in the analysis

Fig. 1: Study population

criteria, and three were missing data. Therefore, a total of 10 patients were included (Fig. 1). The base characteristics of these patients are shown in Tables 1 and 2. The mean APACHE II and SOFA scores were $26.8 \pm 7.9$ and $13.9 \pm 1.7$, respectively. Cardiac output and stroke volume were recorded in six patients: pulmonary artery catheters were used for five and transpulmonary thermodilution method for one. Coronary angiography was performed in two patients and revealed no stenosis.

For the primary outcomes, a MAP increase of more than $30 \%$ was achieved in six patients and a decrease in CAI was observed in five. The effective group was defined as the patient group that achieved at least one of these primary outcomes and consisted of seven patients (Table 3). The noneffective group consisted of the remaining patients who did not achieve at least one of the primary outcomes. Although the overall survival rate on the 28th day was $30 \%$, that on the $3 \mathrm{rd}$ and 7 th day after IABP initiation were $100 \%$ and $70 \%$, respectively, that indicated that the included patients could survive their severe refractory cardiogenic shock with IABP in the acute phase. Stroke volume increased in five of six patients (83\%) who were equipped with pulmonary artery or transpulmonary thermodilution catheter (Table 4). We also performed a subgroup analysis on both the effective and noneffective groups. The results showed that the initial heart rates were lower in the effective group. Furthermore, abnormal heart rhythms were observed in two patients (28.6\%) in the effective group, whereas all three patients in the noneffective group had abnormal heart rhythms.

Table 1: Baseline characteristics of study population

\begin{tabular}{|c|c|c|c|c|c|c|c|c|c|c|}
\hline Case & Age/Sex & Site of infection & $C A I$ & $\begin{array}{l}\text { Corticoster- } \\
\text { oid use }\end{array}$ & $\begin{array}{l}\text { Preload } \\
\text { (CVP, } \mathrm{mm} \\
\mathrm{Hg})\end{array}$ & $\begin{array}{l}\text { Afterload } \\
\text { (MAP, mm } \\
\mathrm{Hg} \text { ) }\end{array}$ & $\mathrm{CO}(\mathrm{L} / \mathrm{min})$ & $\begin{array}{l}\text { Lactate } \\
\text { (mmol/L) } \\
\text { change }\end{array}$ & $\begin{array}{l}\text { Urine out- } \\
\text { put }(\mathrm{mL} / \mathrm{h}) \\
\text { change }\end{array}$ & $\begin{array}{l}\mathrm{CO}(\mathrm{L} / \mathrm{min}) \\
\text { change }\end{array}$ \\
\hline 1 & $47 /$ male & Unknown & 5 & No & 20 & 98 & 1.6 & -11.5 & 15 & 2.2 \\
\hline 2 & $62 /$ male & Respiratory system & 78 & No & 8 & 51 & NA & 1.2 & 140 & NA \\
\hline 3 & $63 /$ male & Respiratory system & 35 & No & 18 & 53 & 1.7 & -0.1 & 5 & 3.8 \\
\hline 4 & $73 /$ male & Respiratory system & 90 & Yes & 15 & 80 & NA & 1.3 & -25 & NA \\
\hline 5 & 76/male & Blood stream & 30 & No & 12 & 90 & 5.1 & 0.0 & -120 & 0.8 \\
\hline 6 & 77/female & Respiratory system & 300 & No & NA & 61 & NA & -8.1 & 60 & NA \\
\hline 7 & 79/male & Respiratory system & 120 & Yes & 13 & 59 & 3.5 & 0.5 & 30 & 2.5 \\
\hline 8 & 79/male & Urinary tract & 5 & Yes & 14 & 53 & 0.8 & 0.1 & -130 & 1.0 \\
\hline 9 & $83 /$ male & Respiratory system & 180 & No & 24 & 41 & 2 & -1.6 & 20 & 1.9 \\
\hline 10 & $84 /$ male & Urinary tract & 100 & Yes & 13 & 83 & 3.5 & 0.3 & 20 & 1.3 \\
\hline
\end{tabular}


Effect of Intra-arterial Balloon Pumping for Refractory Septic Cardiomyopathy: A Case Series

Table 2: Study population: Highlighting of IABP use $(\mathrm{N}=10)$

\begin{tabular}{ll}
\hline & $I A B P$ use $(\mathrm{N}=10)$ \\
\hline Age (mean in years) & $72.3 \pm 11.0$ \\
Male, No. (\%) & $9(90)$ \\
APACHE score & $26.8 \pm 7.9$ \\
SOFA score & $13.9 \pm 1.7$ \\
CAI & $94.3 \pm 86.2$ \\
MAP (mmHg) & $67 \pm 18$ \\
HR (bpm) & $97 \pm 18$ \\
CO (L/min) & $2.18 \pm 1.00$ \\
SV (ml) & $25.1 \pm 13.3$ \\
Lactate (mmol/L) & $9.7 \pm 6.4$ \\
P/F ratio & $171 \pm 81$ \\
Site of infection & \\
$\quad$ Lung (\%) & $6(60)$ \\
$\quad$ Urinary tract (\%) & $2(20)$ \\
$\quad$ Blood (\%) & $1(10)$ \\
$\quad$ Unknown (\%) & $1(10)$ \\
\hline
\end{tabular}

Table 3: Primary outcomes of effective group

\begin{tabular}{lllll}
\hline & \multicolumn{3}{c}{ CAI } \\
\cline { 3 - 5 } & Decrease & No change & Increase \\
\hline \multirow{2}{*}{ MAP } & $\begin{array}{l}\text { Increase of } \geq 30 \% \\
\text { Increase between }\end{array}$ & 3 & 2 & 1 \\
$0-29 \%$ & & 0 & 0 \\
Decrease & 0 & 2 & 1 \\
\hline
\end{tabular}

The three abnormal heart rhythms other than sinus rhythms in the noneffective group consisted of two atrial fibrillations and a series of paroxysmal ventricular contractions accompanied with several ventricular tachycardia. No patients required VA-ECMO in the effective group; however, two eventually required subsequent VA-ECMO due to inadequate cardiac output and multiorgan failure in the noneffective group (Table 5).

\section{Discussion}

To our knowledge, this study is the first case series to report the efficacy of IABP use for cardiogenic shock associated with SCM. In our study, the efficacy of IABP was clarified by MAP increase and CAl decrease in patients whose MAP cannot sustained only by vasoactive agents. Although the mortality rate on 28 th day was $30 \%$ in our study, the death occurred after all of them had survived severe refractory cardiogenic shock in the acute phase. Furthermore, the implementation of VA-ECMO, an extremely invasive treatment, was avoided in these cases. Therefore, we think IABP is an effective strategy for refractory cardiogenic shock in SCM patients.

Selecting appropriate candidates for IABP is essential since it may also decrease MAP in some patients. Based on our results, patients in the noneffective group had higher heart rates than those in the effective group, and all patients in the noneffective group had irregular heart rhythms. These results indicate that low heart rates and regular rhythm, the synchronicity with IABP, are important factors for IABP to be effective in SCM patients. These factors may help determine SCM patients for whom IABP is effective.

Some treatments have been considered for SCM, such as activated protein $\mathrm{C}$, hydrocortisone, NO synthase inhibitor or
Table 4: Primary outcomes of non-effective group

\begin{tabular}{ll}
\hline & IABP use $(N=10)$ \\
\hline Survival & \\
3 day (\%) & $10(100)$ \\
7 day (\%) & $7(70)$ \\
28 day (\%) & $3(30)$ \\
$H R$ & \\
$\quad$ Decrease $\geq 10 \%(\%)$ & $4(40)$ \\
$\quad$ No change (\%) & $2(20)$ \\
Increase $\geq 10 \%(\%)$ & $4(40)$ \\
SV change & \\
$\quad$ No change or decrease (\%) & $1(17)$ \\
$\quad$ Increase $\geq 30 \%(\%)$ & $5(83)$ \\
Required subsequent VA-ECMO & $2(20)$ \\
\hline
\end{tabular}

Table 5: Comparison of effective and non-effective group

\begin{tabular}{lll}
\hline & \multicolumn{2}{c}{ IABP use (N=10) } \\
\cline { 2 - 3 } & $\begin{array}{l}\text { Effective group } \\
(N=7)\end{array}$ & $\begin{array}{l}\text { Non-effective } \\
\text { group }(N=3)\end{array}$ \\
\hline $\begin{array}{l}\text { APACHE score } \\
\text { SOFA score }\end{array}$ & $27.1 \pm 8.5$ & $26.0 \pm 6.5$ \\
Site of infection & $13.6 \pm 1.6$ & $14.7 \pm 1.7$ \\
$\quad$ Lung (\%) & $5(71.4)$ & $1(33.3)$ \\
$\quad$ Urinary tract (\%) & $2(28.6)$ & $0(0)$ \\
$\quad$ Blood (\%) & $0(0)$ & $1(33.3)$ \\
$\quad$ Unknown (\%) & $0(0)$ & $1(33.3)$ \\
Original heart rhythm & & \\
$\quad$ Regular sinus rhythm & $5(71.4)$ & $0(0)$ \\
$\quad$ Irregular rhythm & $2(28.6)$ & $3(100)$ \\
Original HR & $92 \pm 19$ & $107 \pm 9$ \\
Required subsequent VA- \\
ECMO
\end{tabular}

methylene blue, however, no specific treatment could show the clinical efficacy. ${ }^{11,14}$ We also suggest acute blood purification, a less invasive but unconfirmed method, to treat refractory shock. ${ }^{11,13}$ Furthermore, a case series reported $71 \%$ survival after VA-ECMO for refractory SCM. ${ }^{12}$

Although it has not been previously used as a method of mechanical cardiac support, the results of this study suggest that IABP may be a strategy for refractory SCM. Since refractory SCM is a life-threatening condition and there is no evidence-based practice for it, IABP insertion should be considered prior to initiating VA-ECMO when acute blood purification failed to achieve the target MAP.

There are several limitations to this study. First, this is a case series study which include only 10 patients. It is difficult to generalize the results due to the small sample size. Second, since there is no comparison group for the IABP group, it is so unconvincing to claim the efficacy. A following randomized control trial is required to prove our hypothesis, though it seems difficult because of the rarity of candidates. Third, there is no consensus on the definition of SCM, and, therefore, the accuracy of the diagnosis is unclear. Finally, IABP could not provide improvement of long-term survival, in which most clinicians must be most interested. 


\section{ConCLUSION}

Intra-arterial balloon pumping for refractory SCM may be an effective method to stabilize circulation status, especially for patients with low heart rates and regular rhythm.

\section{References}

1. Ehrman RR, Sullivan AN, et al. Pathophysiology, echocardiographic evaluation, biomarker findings, and prognostic implications of septic cardiomyopathy: a review of the literature. Crit Care 2018;22(1):112.

2. Beesley SJ, Weber G, et al. Septic Cardiomyopathy. Crit Care Med 2018;46(4):625-634.

3. Angus DC, van der Poll T. Severe sepsis and septic shock. N Engl J Med 2013;369:840-851.

4. Singer $M$, Deutschman CS, et al. The third international consensus definitions for sepsis and septic shock (Sepsis-3). JAMA 2016;315:801810.

5. Abraham E, Singer M. Mechanisms of sepsis-induced organ dysfunction. Crit Care Med 2007;35:2408-2416.

6. Blanco J, Muriel-Bombín A, et al. Incidence, organ dysfunction and mortality in severe sepsis: a Spanish multicentre study. Crit Care 2008;12(6):R158.
7. Annane D, Vignon $P$, et al. Norepinephrine plus dobutamine versus epinephrine alone for management of septic shock: a randomized trial. Lancet 2007;370(9588):676-684.

8. Mahmoud KM, Ammar AS. Norepinephrine supplemented with dobutamine or epinephrine for the cardiovascular support of patients with septic shock. Indian J Crit Care Med 2012;16(2):75-80.

9. Prabhu SD. Cytokine-induced modulation of cardiac function. Circulation Research 2004;95:1140-1153.

10. Kakihana $Y$, Ito $T$, et al. Sepsis-induced myocardial dysfunction: pathophysiology and management. J Intensive Care 2016;4:22.

11. Sato R, Nasu M. A review of sepsis-induced cardiomyopathy. J Intensive Care 2015;3:48.

12. Bréchot $\mathrm{N}$, Luyt $\mathrm{CE}$, et al. Venoarterial extracorporeal membrane oxygenation support for refractory cardiovascular dysfunction during severe bacterial septic shock. Crit Care Med 2013;41(7): 1616-1626.

13. Nakamura K, Doi K, et al. Endotoxin adsorption by polymyxin $B$ column or intraaortic balloon pumping use for severe septic cardiomyopathy. Am J Emerg Med 2013;31:893.e1-3.

14. Muller-Werdan U, Buerke M, et al. Septic cardiomyopathy-A not yet discovered cardiomyopathy? Exp Clin Cardiol 2006;11(3):226-236. 